\title{
El peso mexicano: la gestión de cobertura del riesgo cambiario mediante la Teoría de los Efectos Olvidados
}

\section{The Mexican Peso: Exchange Risk Coverage Management through the Forgotten Effects Theory}

\author{
Ricardo Salazar-Garza1
}

Received: August 01, 2011

Accepted: February 03, 2012

\begin{abstract}
Resumen
El presente trabajo desarrolla un modelo no lineal de predicción del comportamiento del tipo de cambio a futuro basado en la opinión de los agentes económicos participantes en el mercado dólar/peso. Tales opiniones son tratadas mediante la Lógica Borrosa y una variante de ésta, conocida como la Teoría de los Efectos Olvidados.La finalidad es encontrar un mecanismo de toma de decisiones de cobertura que nos permita una administración de riesgo de tipo de cambio óptima a un menor costo que aquel que conlleva realizar operaciones con los instrumentos de cobertura tradicional. Para el periodo investigado y mediante este modelo, los resultados sustentan que las opiniones conjuntas de los expertos económicos involucrados en la toma de decisiones de administración de riesgo de tipo de cambio brindan mejores resultados que aquellos que utilizan métodos tradicionales en los mercados de futuros.
\end{abstract}

Palabras clave: Tipo de cambio, Lógica Borrosa, expertos en Mercado de Futuros, Efectos Olvidados, Expertones.

\begin{abstract}
This paper is about developing a nonlinear model to predict the behavior of future exchange rate based on the opinion of the economic agents participating in the dollar/peso market. Such views are treated with Fuzzy Logic and a variant of it, known as the Theory of Forgotten Effects. The aim is to find a mechanism for making coverage decisions that allow us an optimal exchange rate risk management at a lower cost than that which involves operations with traditional hedging instruments. For the period of investigation and applying this model, the results support that the collective opinions of economic experts involved in the decision making risk management of exchange rate provide better results than those using traditional methods in the future markets.
\end{abstract}

Kewords: Exchange rate, Fuzzy Logic, Future Markets Experts, Forgotten Effects, Expertones.

1. Ph.D en Administración, Universidad de Monterrey, México.<ricardo.salazar@udem.edu.mx>. 


\section{INTRODUCCIÓN}

El tratar de comprender los movimientos del tipo de cambio ha representado una gran tarea y un importante reto tanto para los académicos como para los investigadores en el área de negocios (Gradojevic, 2002). En el contexto de nuestro país y desde la adopción de un régimen de libre flotación en 1995, el comportamiento del peso mexicano ha estado caracterizado por largos períodos de tranquilidad que repentinamente dan lugar a episodios de alta volatilidad. (Bazdresch \& Werner, 2002).

En este orden de ideas, consideramos que la gestión de cobertura del riesgo cambiario, por parte de los agentes económicos expuestos a apreciaciones o depreciaciones del peso mexicano frente al dólar estadounidense, representa una de las actividades más importantes en el ámbito empresarial. Por eso consideramos que el contar con un mecanismo que permita realizar eficientemente tal gestión de cobertura se ha tornado uno de los objetivos principales de los hombres de negocios mexicanos que interactúan en los mercados internacionales.

\section{Declaración del problema}

Hoy en día, los mecanismos tradicionales de cobertura de riesgo cambiario en el mercado de divisas en general (futuros, forwards), y en el mercado dólar-peso en particular, solo reflejan las condiciones actuales del mercado más la política de tasas de interés de las naciones que están involucradas en el intercambio de las divisas. Sin embargo, no contemplan la influencia que los agentes económicos participantes (traders, brokers) tienen o pueden ejercer sobre el comportamiento y equilibrio del tipo de cambio. Tal situación origina que las acciones de cobertura realizadas por las entidades que asumen riesgos de tipo de cambio en sus operaciones de negocio sean demasiado costosas e inefectivas.

\section{Objetivo general}

El objetivo es construir un modelo de gestión no lineal de cobertura de riesgo cambiario que pronostique la evolución futura del tipo de cambio de equilibrio, basado en el desempeño esperado de las variables macroeconómicas que inciden en el mismo, apoyado en una variante de la Lógica Borrosa conocida como la Teoría de los Efectos Olvidados. Dicho modelo estará sustentado en el análisis, estudio e interpretación del comportamiento de participantes expertos (traders, brokers) en el mercado de divisas, y sus reacciones ante cambios en las variables que inciden en el equilibrio del tipo de cambio peso-dólar. Con ello se busca que la gestión de riesgo de tipo de cambio maximice la eficiencia y minimice el costo de una cobertura respecto de los resultados que se pueden obtener al utilizar los procesos de cobertura tradicionales que existen en los mercados de divisas.

\section{REVISIÓN DE LA LITERATURA}

Como resultado de la crisis del peso mexicano de diciembre de 1994, el Banco de México se vio forzado a abandonar su régimen de flotación predeterminado y adoptar un régimen de libre flotación del peso frente a las demás monedas del mundo (Ortiz, 2004). Hasta el mes de noviembre de 1994, los agentes económicos contaban con información acerca del comportamiento del tipo de cambio en el futuro. En efecto, el Banco de México trazaba el tipo de cambio deseado en un horizonte de tiempo dado, lo comunicaba a los agentes económicos, y orientaba su política monetaria hacia la obtención de ese tipo de cambio objetivo. Desde el año 1989 hasta el año 1994 el tipo de cambio funcionaba como un "ancla nominal de la economía" (Elizondo \& Sepúlveda, 2004).

Después de la crisis citada, varias hipótesis se formularon con la intención de encontrar una explicación a este fenómeno. Estas hipótesis fueron debatidas por Francisco Gil Díaz y Agustín Carstens durante el evento Annual January ASSA Meeting, que tuvo lugar en San Francisco, Estados Unidos de América, en enero de 1996 (Gil \& Carstens, 1996).

Las hipótesis se estructuraban sobre la base de que una moneda sobrevaluada, un crédito bancario laxo, la falta de información del banco central hacia los agentes sobre la situación real de la economía del país y un síndrome de endeudamiento excesivo habían ocasionado 
la crisis mexicana. En su exposición, Gil y Carstens concluyeron que un tipo de cambio semifijo, una alta disponibilidad de capitales a corto plazo - los cuales podían ser convertidos a dólares con facilidad y ser retornados a su país de origen-, así como un elevado número de acontecimientos políticos negativos durante el año de 1994, habían sido las causas fundamentales de la devaluación del peso.

A partir de estas conclusiones, en relación al tipo de cambio del peso mexicano, resulta importante enfocarse sobre la hipótesis de que un tipo de cambio sobrevaluado y la conclusión de una problemática derivada de un tipo de cambio semifijo fueron factores que ocasionaron incertidumbre en el mercado de divisas mexicano. Estos factores influenciaron al grado de que, por ejemplo, en 1995 la cotización del dólar frente al peso, llegó a oscilar de MXP \$5.3625 el 8 de febrero hasta MXP \$7.5875 pesos por dólar el 10 marzo. Ante estos acontecimientos, y el hecho de que el tipo de cambio a futuro era ya un fenómeno incierto, surgió la necesidad de retomar las teorías sobre los tipos de cambio para modelar el comportamiento esperado del peso mexicano frente al dólar estadounidense y fundamentar una idea general de equilibrio bajo un contexto de libre flotación.

Frente a esto surge la problemática derivada de los estudios de Messe y Rogoff (1983) así como de Backus (1984). Dichos autores argumentaron que la evolución del tipo de cambio seguía un modelo simple de "paseo aleatorio", descartando con ello la utilidad de los modelos clásicos como "la paridad del poder adquisitivo" desarrollada por David Ricardo en el siglo XIX, y la "Teoría de paridad cubierta de intereses" desarrollada por Keynes en esa misma época, así como el "Modelo de flujo con movimientos de capitales" desarrollado por Mundell y Flemming en los años sesenta del siglo pasado, entre otros.

Posterior a los estudios de Messe y Rogoff, las investigaciones orientadas a tratar de explicar el comportamiento del tipo de cambio sobre la base de modelos económicos fueron perdiendo adeptos. Tal es el caso del estudio realizado por De Grauwe, Dewachter y Embrechts (1993), en el que manifiestan que:

los participantes del mercado cambiario se hicieron escépticos sobre la utilidad de los modelos económicos, lo que modificó el rumbo de las investigaciones (Prefacio).

Las nuevas investigaciones se centraron en estudiar el comportamiento de los agentes económicos que interactuaban en los mercados de divisas, (traders, brokers, etc). Por su parte, Goldberg y Tenorio (1997) mencionan que:

...en verdad, dado el alto nivel de participantes en el mercado de divisas, interpretando tanto información pública como privada, resulta natural el asumir que las expectativas de equilibrio del tipo de cambio se forman a partir de aspectos microeconómicos fundamentales así como variables de estructura macroeconómica. (p. 305)

entendiendo por aspecto fundamental la manera en la que los agentes interpretan, reaccionan y toman decisiones basadas en el comportamiento esperado de estas variables.

A este respecto, Lyons y Evans (2002) y Yao (1997) opinan que en años recientes ha existido gran evidencia de que el comportamiento de los operadores de divisas (traders) y otros participantes del mercado puede influenciar el equilibrio de los tipos de cambio. Este tipo de estudios originaron la creación de una nueva línea de investigación, esto es, el estudio del comportamiento y el análisis de la interpretación de los agentes que participan en el mercado (expertos), así como la importancia que ellos pudieran otorgar a las variables tanto macro como microeconómicas que, en opinión de los expertos,influencian en los tipos de cambio (Wu \& Goo, 2005; Gradojevic, 2002).

La nueva línea de investigación se basa en el estudio del comportamiento de los participantes del mercado cambiario y en sus reacciones ante cambios en las variables que inciden en el tipo de fluctuación de equilibrio. A partir de ella se intenta determinar lo siguiente:

a. Si existe una relación de causalidad entre el comportamiento de las variables macroeconómicas y el tipo de cambio de equilibrio que validen una relación causa-efecto.

b. Si los participantes expertos del mercado cambiario inciden directamente en el tipo de cambio 
de equilibrio, más allá del comportamiento de las variables macroeconómicas mismas.

c. Si es posible aplicar eficientemente un modelo de gestión de cobertura de riesgo de tipo de cambio que involucre nuevas líneas de investigación como la de la lógica borrosa, y otras teorías. Tal es el caso de la de los efectos olvidados que nos permitirían obtener un mecanismo de cobertura de riesgo con mejores resultados.

d. Si los resultados obtenidos por este nuevo modelo de gestión propuesto son mejores que aquellos que se pueden obtener mediante la utilización de modelos de cobertura tradicional y que implican la utilización del comportamiento histórico del tipo de cambio de equilibro.

Actualmente las empresas se involucran en actividades que incluyen el intercambio de un número muy variado de divisas de los diferentes países con los que realizan comercio, quedando expuestas a los movimientos que los tipos de cambio de estas divisas puedan sufrir en el tiempo en el que los negocios están siendo desarrollados. El riesgo cambiario representa la posibilidad de que los flujos futuros de una compañía (derivados de operaciones en monedas distintas a la nacional) puedan verse alterados por fluctuaciones no esperadas en el tipo de cambio (Rupeika-Apoga, 2005). En los mercados internacionales, la forma más común de proteger los flujos futuros de efectivo contra el riesgo cambiario es mediante la adquisición de contratos forward. Según Hull (2002), este tipo de contratos ha sido definido como: “... un acuerdo para comprar o vender un activo (divisa) a una fecha futura a un precio determinado pactado con anticipación" (p. 4).

El contrato forward, al igual que los contratos de futuros, tiene la desventaja de que solo refleja las condiciones actuales del mercado, así como la política de tasas de interés de las naciones que están involucradas en el intercambio de las divisas. Ello ocasiona que generalmente la cobertura sea demasiado costosa. $\mathrm{Si}$ finalmente una compañía está asumiendo el riesgo cambiario sobre la divisa de algún país con altas tasas de interés, la diferencia (spread) entre la divisa doméstica y la extranjera será tal que ocasionará que el tipo de cambio futuro se torne considerablemente alto. Ante la eventualidad del alto costo de la cobertura, muchas empresas toman la decisión de no cubrirse y prefieren correr un riesgo cambiario que muchas veces resulta en grandes pérdidas por variaciones no deseadas en el tipo de cambio (Rupeika-Apoga).

Según Murray (2005) y Rupeika-Apoga, la incertidumbre es una constante que no se ha podido eliminar con los instrumentos de cobertura tradicional desarrollados en los mercados financieros. Por esto los investigadores han buscado alternativas que permitan tratar tal incertidumbre orientándose a estudiar otras áreas del comportamiento de los mercados de cobertura; en otras palabres, ahora se enfocan en analizar las características, las acciones y reacciones de aquéllos que interactúan diariamente en estos mercados: los traders y los brokers de divisas.

El tratamiento de la incertidumbre en el ámbito de las ciencias sociales ha utilizado la Lógica Borrosa para tratar de captar el sentimiento de los participantes del mercado cambiario sobre el comportamiento del tipo de cambio. Sin embargo, los estudios realizados han sido escasos y se han limitado a intentar pronosticar el comportamiento de divisas como el dólar canadiense y el dólar taiwanés, ambas frente al dólar americano, (Wu \& Goo, 2005; Gradojevic, 2002)). Tales estudios se han conducido a través de una variante de esta Lógica Borrosa conocida como Redes Neuronales Artificiales (RNA). Hasta la fecha no existe evidencia de trabajos realizados para desarrollar modelos de cobertura de riesgo cambiario utilizando la teoría de los Efectos Olvidados; tampoco existen aplicaciones de este tipo orientadas al mercado de divisas mexicano y al tratamiento de su moneda, el peso.

Las Redes Neuronales Artificiales son una clase general de modelos no lineales que han sido exitosamente utilizados para resolver una amplia gama de problemas, tales como diagnósticos médicos, selección de productos, sistemas de control, reconocimiento de patrones y pronósticos. Las aplicaciones financieras más importantes se han desarrollado en los campos de opciones, predicciones de bancarrota, predicciones de movimientos en el mercado de acciones y en pronósticos de tipos de cambio (Gradojevic, 2002).

En contraste con las redes neuronales artificiales, la teoría de los Efectos Olvidados siempre mantiene 
actualizadas las decisiones de los expertos, así como las relaciones causa-efecto entre las variables que el mismo experto ha señalado, a fin de ponderar, en un contexto global, todas las decisiones que han venido afectando a un problema en particular. Asimismo, a diferencia de las redes neuronales artificiales —en las que la información se va sustituyendo por aquella que incorpora los resultados basados en la información más reciente- - la teoría de los efectos olvidados considera todo el espectro de decisiones tomadas en función a la percepción del comportamiento futuro de alguna variable, y las pondera para tratar de no olvidar la importancia que puede tener una variable en un contexto en particular.

La principal diferencia entre una red neuronal y la teoría de los Efectos Olvidados es que la primera utiliza información histórica, mientras que la segunda refleja el posible tratamiento de una variable sobre la base del comportamiento esperado a futuro de la misma; tal comportamiento está sustentado en la opinión de un experto o de un grupo de ellos.

\section{METODOLOGÍA}

\section{Lógica Borrosa}

La palabra "borroso" significa algo que no es claro; es decir, es algo que no todos entiende por igual o, en su defecto, que existen varias maneras de entenderlo. También puede significar que no existe una respuesta concreta, que tal respuesta puede no tener solamente uno o dos valores, sino que la lógica borrosa alcanza a ver lo que existe entre dos extremos. (Bojadziev \& Bojadziev, 1997).

Bojadziev y Bojadziev indican que las matemáticas clásicas se basan en respuestas específicas. En cambio, la lógica borrosa busca más resultados posibles entre los dos valores extremos que las matemáticas clásicas ofrecen para entender conceptos con razonamiento humano. Fung (2005) establece la Teoría Borrosa como un elemento para representar claramente lo que puede parecer una idea vaga o imprecisa de los conceptos comunes que se utilizan en el lenguaje natural humano. Esta teoría es definida como una parte extendida de los conjuntos clásicos capaz de formar la imprecisión propia de las intuiciones de las personas.

\section{Subconjuntos Borrosos}

El origen de la teoría de subconjuntos borrosos se remonta a 1965 con la definición según Lofti Zadeh (1972) de "conjunto borroso". Zadeh menciona que los subconjuntos borrosos son un paso hacia el acercamiento entre la precisión de las matemáticas clásicas y la sutil imprecisión real. En el año 1995 Kosko menciona que sí es posible concebir el pensamiento como un subconjunto borroso y no como un lenguaje de caracteres, ya que la borrosidad reconoce un grado de pertenencia para cada elemento. Esto es, utilizar un número real entre 0 y 1 , lo cual resulta más simple y mucho más cercano a la manera hábil de pensar del hombre, al rehabilitar el subjetivismo y la imprecisión.

Los conjuntos borrosos representan el conocimiento humano describiendo objetos o situaciones en términos imprecisos. Así como el conocimiento humano tiene razonamiento, según esta teoría las observaciones tienen más de dos estados lógicos (Varela, 2001). Una extensión de los subconjuntos borrosos son "los expertones"1, concepto cuyo desarrollo se debe a Kaufmann y Gil-Aluja (1986). Estos "expertones" permiten ver la opinión de varios expertos respecto de un tema, libres para proporcionar su decisión en intervalos de confianza.

Un ejemplo sería pedir a un experto si cree que la incidencia de inflación será en el tipo de cambio. En su respuesta se le dará varias alternativas entre " 0 " si cree que no afecta en lo absoluto o "1" si afecta mucho, pasando por los rangos intermedios. Al contestar de esta manera, se observa que el experto no tiene que basar su respuesta en dos alternativas (cero o uno), sino que puede utilizar los rangos intermedios de dichas respuestas para otorgar un resultado más específico. Si la encuesta se entrega a más expertos y se recoge dicha información, se puede llegar a un resultado más exacto.

1 El término "expertón” se define más adelante en el artículo. 
Las variables de incidencia en el tipo de cambio de equilibrio

Para predecir el tipo de cambio, los modelos estructurales requieren primero determinar el valor de sus variables explicativas (Cortez, 2004,). Las variables de esta investigación están divididas en dos grupos:

- Variables domésticas: representan en su conjunto los indicadores más relevantes de la economía mexicana que impactan el tipo de cambio peso-dólar.

- Variables externas: es elconjunto de indicadores económicos - tanto de los Estados Unidos de Norteamérica como del resto del mundo- que impactan de manera directa la cotización del peso frente al dólar estadounidense.

Para validar las variables descritas se realizó una revisión de la literatura enfocándonos en los modelos de pronóstico y determinación del tipo de cambio, y así analizar cuáles variables han sido ya utilizadas mediante otras metodologías con el mismo objetivo de estudio. A partir de la aplicación de pruebas estadísticas — como las de esfericidad de Barlett y Test K-M-O (KaiserMeyer-Olkin) — se determina la relación existente entre dichas variables y el tipo de cambio peso-dólar, aspecto fundamental para nuestra investigación.

\section{Los expertos y las variables de incidencia}

Se considera que un experto es una persona que tiene amplio conocimiento y experiencia en una actividad o campo de una técnica específica. Estos expertos ofrecen su pericia y juicio para fundamentar las teorías y situaciones dotándolas de credibilidad (Tichy, 2002). Desde esta perspectiva, ellos están en capacidad de interpretar correctamente las informaciones sobre dicho campo de actividad y ofrecen evaluaciones mucho más específicas, concretas y reales sobre las posibles teorías que se están estudiando y/o evaluando. Con el fin de entender la evidencia o determinar el problema de un hecho, los expertos se basan en sus estudios y los fundamentan con experiencia y conocimiento.

Dicho lo anterior, es necesario determinar quiénes son los expertos y, por lo tanto, se deben conocer las características específicas que deben cumplir cada uno de ellos. En el estudio Reliability and the Admissibility of Experts, Dale A. Nance (2002) considera que se le puede nombrar "experto" a una persona que tiene habilidades, entrenamiento y una educación altamente avanzada y especializada sobre teorías e investigaciones. La confianza que los expertos proyectan se debe a su asumida habilidad de saber interpretar cómo reaccionarán los diversos recursos económicos, políticos y sociales, para así llegar a sus metas finales (Tichy, 2002).

El proceso que un experto lleva acabo para evaluar, dictaminar y ofrecer una opinión se basa en la vigilancia de las variables observadas a partir de un análisis de la información. Después de valorar la importancia de estas variables, convierte su visión acerca del comportamiento de las mismas en decisiones de negocio o en apuestas direccionales de mercado. En el contexto de esta investigación, el experto que se determinó como elegible para ser encuestado reunía las siguientes calificaciones:

A. Ser miembro activo del sistema financiero mexicano. En otras palabras, laborar en una institución financiera: banco, casa de bolsa, casa de cambio o empresa de corretaje.

B. Tener una posición ejecutiva, ya sea en Dirección, Subdirección, Senior Trader o Broker para la empresa donde labora.

C. Trabajar en el área de divisas de la institución a la que pertenece, ya sea en el mercado spot o en el área de productos derivados financieros de la institución.

D. Tener como mínimo cinco años de experiencia en la operación de divisas en el mercado interbancario.

E. Contar con experiencia en el manejo de la volatilidad de tipos de cambio en situaciones de crisis financieras.

En el Anexo A de esta investigación se puede observar el documento oficial donde los expertos de Base Internacional Casa de Bolsa y Banregio señalan el conjunto de variables que, en su opinión, inciden en el comportamiento del tipo de cambio de equilibrio: 
A. Variables macroeconómicas y micro económicas domésticas del mercado de divisas mexicano que, en opinión de los expertos, inciden en la determi- nación del tipo de cambio de equilibrio peso-dólar (Tabla 1).

Tabla 1.

Variables Domésticas del Mercado de Divisas Mexicano.

\begin{tabular}{cll}
\hline Nombre & \multicolumn{1}{c}{ Variable } & \multicolumn{1}{c}{ Label } \\
\hline VAR0001 & $\begin{array}{l}\text { Reservas Internacionales de Banxico. } \\
\text { Monto en USD de subastas diarias realizadas por Banxico como } \\
\text { Consecuencia de excedentes en el ingreso por venta de petróleo. }\end{array}$ & $\begin{array}{l}\text { Reservas Internacionales } \\
\text { Subastas USD }\end{array}$ \\
VAR0003 & $\begin{array}{l}\text { Tasa de interés Cetes 28 días. } \\
\text { IARdice de Precios al Consumidor. }\end{array}$ & CETES \\
IÁndice Precios \\
VAR0005 & Índice subyacente de precios al consumidor (excluye los elementos & Índice subyacente de Precios \\
& volátiles de la canasta básica). & Bonos mexicanos \\
VAR0006 & Mercado de Bonos Domésticos. & IPC BMV \\
VAR0007 & Índice de Precios y Cotizaciones de la Bolsa Mexicana de Valores. & Mercados Futuros USD \\
VAR0008 & Mercado de Futuros del MXN operados en Mex Der. & Balanza Comercial \\
VAR0009 & Balanza Comercial. & Desempleo \\
VAR0010 & Tasa de desempleo. & PIB \\
VAR0011 & PIB. & Política Monetaria \\
VAR0012 & Política monetaria emitida por el Banco de México. & Petróleo y Gas \\
VAR0013 & Precios de los energéticos (Petróleo, Gas). & Producción industrial \\
VAR0014 & Índice de producción industrial. & Remesas \\
VAR0015 & Volumen de remesas familiares al país. & \\
\hline
\end{tabular}

B. Variables macroeconómicas y micro económicas externas del mercado de divisas mexicano que, en opinión de los expertos, inciden en la determina- ción del tipo de cambio de equilibrio peso-dólar (Tabla 2).

Tabla 2.

Variables Externas del Mercado de Divisas Mexicano.

\begin{tabular}{|c|c|c|}
\hline Nombre & Variable & Label \\
\hline VAR0001 & Datos de empleo (Non Farm Payrolls y tasa de desempleo). & Non Farms Payroll \\
\hline VAR0002 & Tasas de interés. & Tasa de Interes \\
\hline VAR0003 & Minuta de la junta del $F O M C$ del $F E D$. & Minuta $F E D$ \\
\hline VAR0004 & Beige Book. & Beige Book \\
\hline VAR0005 & Flujo neto de Capital (tenencia de Treasuries por extranjeros, TIC data). & Total Net TIC FLOWS \\
\hline VAR0006 & Balanza Comercial. & Balanza Comercial \\
\hline VAR0007 & $\begin{array}{l}\text { Inflación (PPI y CPI subyacente muy importante en ambos) } \\
\text { (PCE Price Index). }\end{array}$ & Inflación \\
\hline VAR0008 & $\begin{array}{l}\text { ISM Manufacturero y No Manufacturero (estado de la economía, } \\
\text { lecturas mayores o menores de } 50 \text { ). }\end{array}$ & ISM Manufacturero \\
\hline VAR0009 & Cuenta Corriente. & Cuenta Corriente \\
\hline VAR0010 & Ventas al detalle. & Adjusted Retail Sales \\
\hline VAR0011 & Pedidos de bienes duraderos. & Durable Goods Services \\
\hline VAR0012 & Producción industrial. & Industrial Production \\
\hline VAR0013 & $G D P$. & GDP Billion (Nominal USD) \\
\hline VAR0014 & Commitment of traders IMM (USD/MXN). & $I M M(\mathrm{UDS} / \mathrm{MXN}$ \\
\hline VAR0015 & Ventas de casas: nuevas, existentes. & New Home Sales \\
\hline VAR0016 & Mercado de Futuros del MXN operado en CME. & Futuros del MXN en CME \\
\hline VAR0017 & $\begin{array}{l}\text { Tipos de cambio de las monedas de países con economías emergentes: } \\
\text { (BRL, ZAR, TWD). }\end{array}$ & T/C Países emergentes \\
\hline VAR0018 & $\begin{array}{l}\text { Tipos de cambio de las monedas de los países del G7 (EUR, USD, } \\
\text { CAD, GBP, JPY, AZD, CHF). }\end{array}$ & T/C Países G7 \\
\hline
\end{tabular}




\section{MODELO}

Para el desarrollo de esta investigación se construyó un modelo no lineal de gestión de cobertura de riesgo de tipo de cambio, el cual se divide en cuatro etapas. En la primera etapa, denominada de insumos, se recabó la información económica que proporcionaron los expertos que participan en el desarrollo del proyecto. Dicha información será sustentada teóricamente.

Sobre la base de esta información se solicitará a los expertos que determinen las variables - tanto domésticas como externas - que en su opinión inciden en el comportamiento del tipo de cambio.Tales variables deberán ser validadas con un estudio de correlación que soporte el argumento de incidencia manifestado por dichos expertos. De forma paralela, haremos un estudio de la evolución histórica del tipo de cambio, a fin de sentar las bases para el estudio comparado que vamos a realizar, utilizando el ratio de cobertura de varianza mínima, comúnmente conocido como Hedge Ratio.

En la siguiente etapa, denominada de proceso, iniciamos con una segunda encuesta donde solicitamos al experto que nos indique el impacto esperado en cada una de las variables determinadas en la etapa anterior sobre la variable tipo de cambio. Una vez obtenidos los resultados de esta segunda encuesta, se procederá a procesar los datos mediante la utilización de lógica borrosa, para construir los expertones y determinar los efectos olvidados. A partir de este punto construiremos una ecuación de cobertura, a la que denominaremos "Fuzzy Hedge", y que incluirá aquellas variables que en opinión de los expertos son relevantes para el período en estudio. Una vez determinada la ecuación se realizará una tercera encuesta donde se preguntará al experto acerca de su percepción sobre el desempeño o evolución de cada una de las variables de la ecuación en el período evaluado. Con los datos obtenidos se procederá a calcular el $\mathrm{Z}$ valor, que nos indicará el porcentaje de nuestra exposición de riesgo de tipo de cambio que habremos de cubrir.

De forma paralela, realizaremos los cálculos de varianza sobre la evolución histórica del tipo de cambio, para preparar el inicio del estudio comparado. En esta tercera etapa, denominada de acción/evaluación, se tomarán las decisiones de cobertura de riesgo de tipo de cambio de acuerdo con los criterios de aplicación del Z valor. Simultáneamente se determinarán los niveles de cobertura en el estudio comparado y se realizarán los cálculos sobre la Hedge Ratio, así como las opciones de estar totalmente descubierto o cubierto. Se recabaron datos durante un período de doce meses en bloques trimestrales; se decidió considerar el año 2008 por ser un sinodal importante para el modelo, esto debido a la crisis que se desató durante ese período.

En la última etapa, analizaremos los resultados y se estimará el costo total de la cobertura utilizando nuestra ecuación denominada Fuzzy Hedge. Asímismo, calcularemos el resultado obtenido mediante la utilización de la Hedge Ratio que estamos considerando evaluar en la investigación. En el Anexo B se observa una representación gráfica del modelo desarrollado.

\section{Construcción de un Expertón}

Un expertón es el resultado de una agrupación de ideas de expertos basadas en un tema que tenga borrosidad, incertidumbre o muchas posibles respuestas dependiendo de las personas. Cada experto tiene una opinión y cada una de sus respuestas son válidas pues están basadas en sus experiencias y estudios. Sin embargo, no todos creen lo mismo, pues siempre hay una cierta subjetividad en sus respuestas. Entonces agrupándolas siguiendo cierta metodología se podrá llegar a una respuesta precisa. Para obtener una respuesta concisa de opiniones borrosas se requiere combinar las opiniones de varios "expertos" encuestados o interrogados para así llegar, mediante un modelo basado en la lógica borrosa, a una respuesta más exacta.

En este sentido, el expertón reúne el conocimiento de todos los expertos sobre un tema dado; es decir, a partir de un expertón se comienza a resolver temas específicos sobre los que ellos tienen un amplio grado de experiencia, juicio, comprensión y dominio, aunque difieran en sus opiniones. Esto se hace con el fin de aclarar cuestiones en las que se tiene un alto nivel de subjetividad. Entonces, se denomina expertón a una respuesta basada en el conjunto de los conocimientos de varios expertos en un tema estudiado.

Un ejemplo para describir cómo se construye un expertón sería el siguiente: se buscan expertos en el 
área de mercado de divisas. Estos expertos son encuestados sobre las variables que en su opinión inciden en la determinación del tipo de cambio y se obtiene las siguientes variables:

- Tasas de interés

- Riesgo País

- Inflación

- Balanza Comercial

Para efectos de mostrar cómo es el proceso, tomaremos tres de ellas:

$$
\begin{aligned}
& a=\text { tasas de interés externas } \\
& b=\text { inflación } \\
& c=\text { riesgo país }
\end{aligned}
$$

variables que se referencian como:

$$
E=\{a, b, c\}
$$

La opinión de cinco expertos sobre la importancia de las variables a, b, y c, del fenómeno "equilibrio del tipo de cambio" se expresa a través de números borrosos como se muestra en la Tabla 3. Los mismos expertos califican, conforme a su intuición, el nivel de incidencia de cada una de las variables sobre el tipo de cambio para posteriormente hacer una matriz de resultados.

Los valores que muestran son números entre el $0 \mathrm{y}$ el 1; el número 1es el que más incide y el número 0 el que menos incide en el tipo de cambio.

\section{Encuesta para medición de impacto y tendencia en las variables}

La encuesta de impacto es un tipo de medición que se puede utilizar para responder el grado en que cada variable influye al tipo de cambio así como su valor de incidencia.

Tabla 3.

Explicación de una Matriz Borrosa de Resultados

\begin{tabular}{cccc}
\hline Expertos & Variable A & Variable B & Variable C \\
\hline 1 & 0.3 & 0.6 & 0.2 \\
2 & 0.7 & 0.4 & 0.9 \\
3 & 0.1 & 0.1 & 0.4 \\
4 & 0.4 & 0.6 & 0.8 \\
5 & 0.2 & 0.4 & 0.6 \\
\hline
\end{tabular}

Tabla 4.

Valores de Incidencia: Encuesta de Impacto

\begin{tabular}{cl}
\hline Valor & \multicolumn{1}{c}{ Incidencia } \\
\hline 0 & sin incidencia \\
0.1 & prácticamente sin incidencia \\
0.2 & casi sin incidencia \\
0.3 & muy débil incidencia \\
0.4 & débil incidencia \\
0.5 & mediana incidencia \\
0.6 & incidencia sensible \\
0.7 & bastante incidencia \\
0.8 & fuerte incidencia \\
0.9 & muy fuerte incidencia. \\
1 & la mayor incidencia \\
\hline
\end{tabular}

En la Tabla 4 se puede identificar que las respuestas indicarían el nivel de incidencia que se tiene para cada variable contra el tipo de cambio. Sin embargo, también se puede establecer una respuesta con un nivel de verdad de una proposición.

\section{Encuesta de Tendencia}

En esta encuesta se pregunta a un par de expertos alternos. Ambos deben contestar la manera en que estiman el movimiento de las variables estudiadas. Los posibles resultados serían los siguientes:

Tabla 5.

Ejemplo de Posibles Resultados para una Encuesta de Tendencia

\begin{tabular}{cl}
\hline Valores & \multicolumn{1}{c}{ Resultados } \\
\hline 0.00 & Muy negativo \\
0.25 & Negativo \\
0.50 & Estable \\
0.75 & Positivo \\
1.00 & Muy positivo \\
\hline
\end{tabular}

Grado de incidencia que es asignado a cada variable entre el 0 y 1 
Cada uno de estos resultados de tendencia tiene un valor específico que da un resultado exacto para tomar la decisión final en la ecuación de cobertura que se pretende construir. En ese sentido, por ejemplo, la respuesta de uno de los expertos a la pregunta cómo esperan los movimientos de las variables estudiadas podría ser lo siguiente:

Tabla 6.

Ejemplo de resultados para la encuesta de tendencia

\begin{tabular}{cccc}
\hline Experto & $\mathrm{A}$ & $\mathrm{B}$ & $\mathrm{C}$ \\
\hline 1 & Negativo & Positivo & Estable \\
\hline
\end{tabular}

Tabla 7.

Nivel de incidencia del ejemplo en Tabla 6

\begin{tabular}{cc}
\hline Variable & Incidencia \\
\hline A & 0.25 \\
B & 0.75 \\
C & 0.50 \\
\hline
\end{tabular}

\section{La determinación de los efectos olvidados}

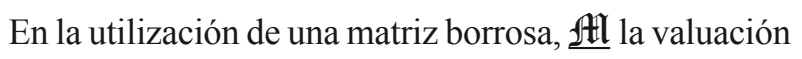
de un par:

$$
\left(X_{i}, X_{j}\right) \in R \subset(A * B)
$$

en donde $A$ y $B$ son referenciales dados que en lugar de tomar un valor entre 0 y 1 pueden tomar un valor entre 0 y 1 , expresado de la siguiente manera:

$$
\left(X_{i}, X_{j}\right) \in \underline{\mathfrak{A l}} \quad v\left(x_{i}, x_{j}\right) \in(0,1)
$$

En el supuesto de considerar solamente la incidencia entre un grupo de causas que a la vez son efectos unos con otros, vamos a realizar operaciones con la misma matriz borrosa. Tal es el caso de las variables que influyen en el tipo de cambio: ellas inciden en este, tanto como entre ellas mismas. Por ser aspectos de índole microeconómica y macroeconómica, la relación causa efecto se torna muy dinámica, de tal manera que se imposibilita determinar cuáles de las variables podrían ser consideradas como causas y cuáles como efectos.
Veamos un ejemplo con la matriz borrosa de sólo una muestra de variables domésticas que en opinión de los expertos inciden en el comportamiento del tipo de cambio peso-dólar.

En la Tabla 8 se presentan las valuaciones de las incidencias proporcionadas por los expertos. Se observa que las reservas (1) tienen una influencia sobre el mercado de bonos domésticos de 0.8 . Esto constituye un factor importante para el mercado de bonos, pero no es el único. Podemos ver que los CETES a 28 días (3) y los futuros del peso (8) tienen la incidencia de mayor importancia (1) sobre el mercado de bonos.

Según se observa de lo anterior, los expertos han dado sus valuaciones para las incidencias directas; tendremos entonces que calcular los efectos de segundo orden de la siguiente manera:

$$
\mathfrak{A l l}_{(2)}=\mathfrak{A l}_{(1)} \text { o } \underline{\mathfrak{A l}}_{(1)}
$$

Si una matriz $\underline{\mathfrak{A l}}$ es reflexiva, es decir que su diagonal se halla formada por 1's, las valuaciones $\underline{\mathfrak{A l}} * \mathfrak{A H}$ son siempre más grandes o iguales a las de $\mathfrak{A t l}$; en otras palabras.

$$
\underline{\mathfrak{A l}} \subset \underline{\mathfrak{A l}} \circ \underline{\mathfrak{A l}}
$$

y, por ende, podemos realizar:

$$
\underline{\mathfrak{A l}} \circ \underline{\mathfrak{A l}}-\underline{\mathfrak{A l}}
$$

para con ello eliminar los efectos de primer orden e identificar exclusivamente los de segundo orden o efectos olvidados (ver Tabla 9).

Se calcula primero el cuadrado de la matriz de referencia de las opiniones de los expertos y posteriormente se estima lo siguiente:

$$
\underline{\mathfrak{A l}}_{(2)}=\underline{\mathfrak{A l}}_{(2)}-\underline{\mathfrak{A l}}_{(1)}
$$

Ahora analizamos los efectos de segunda generación o efectos olvidados por los expertos y encontramos que:

$(9 \rightarrow 12)$ El mercado de bonos ejerce gran influencia sobre el tipo de cambio, 
Tabla 8.

Ejemplo con la Matriz de Variables Domésticas

\begin{tabular}{|c|c|c|c|c|c|c|c|c|c|c|c|c|}
\hline & 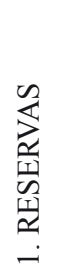 & 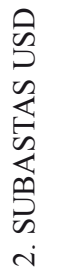 & 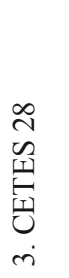 & $\begin{array}{l}\circlearrowright \\
\triangleq \\
\dot{\sigma}\end{array}$ & 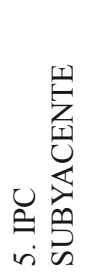 & 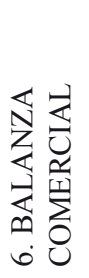 & $\begin{array}{l}\sum_{n} \\
U \\
\vdots \\
i\end{array}$ & 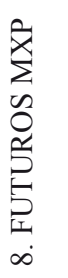 & 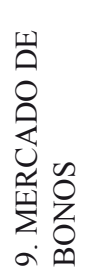 & 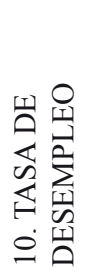 & $\begin{array}{l}\stackrel{\varrho}{a} \\
\doteq \\
\doteq\end{array}$ & 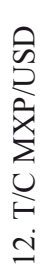 \\
\hline 1. RESERVAS & 1 & 0.02 & 0.9 & 0.8 & 0.1 & 0.5 & 0.1 & 0.5 & 0.8 & 0.2 & 0.3 & 0.6 \\
\hline 2. SUBASTAS USD & 0 & 1 & 0.3 & 0.9 & 0.8 & 0.6 & 0.5 & 0.7 & 0.6 & 0.8 & 0.5 & 1 \\
\hline 3. CETES 28 & 0.1 & 0.4 & 1 & 0.8 & 0.1 & 0.1 & 0.3 & 0.2 & 1 & 0.2 & 0 & 0.1 \\
\hline 4. IPC & 0 & 0.6 & 0.1 & 1 & 0.4 & 0.2 & 0.1 & 0.1 & 0.2 & 0 & 0 & 0.4 \\
\hline 5. IPC SUBYACENTE & 0 & 1 & 0.3 & 0.8 & 1 & 1 & 0.8 & 0.3 & 0.5 & 0.2 & 0.2 & 0.4 \\
\hline 6. BALANZA COMERCIAL & 0.2 & 0.3 & 0.4 & 0.6 & 0.5 & 1 & 1 & 1 & 0.8 & 1 & 1 & 1 \\
\hline 7. IPC BMV & 0.3 & 0.2 & 0.2 & 0.1 & 0 & 0.3 & 1 & 0.2 & 0.8 & 0.4 & 0.3 & 0.8 \\
\hline 8. FUTUROS MXP & 0.2 & 0 & 0.1 & 0 & 0 & 0.2 & 1 & 1 & 0.9 & 1 & 0 & 0.6 \\
\hline 9. MERCADO DE BONOS & 0.2 & 1 & 0.3 & 1 & 0.3 & 0.3 & 0.5 & 0 & 1 & 0.3 & 0.1 & 0 \\
\hline 10. TASA DE DESEMPLEO & 0.1 & 0.8 & 0.2 & 0.3 & 0 & 0 & 0.8 & 0.6 & 0.2 & 1 & 0.2 & 0.4 \\
\hline 11. PIB & 0 & 0.3 & 0 & 0.1 & 0 & 0.2 & 0.3 & 0.2 & 0.3 & 0.3 & 1 & 0.3 \\
\hline 12. T/C MXP/USD & 0 & 0.8 & 0.1 & 0 & 0.1 & 1 & 0.6 & 0.5 & 0 & 0.2 & 0.1 & 1 \\
\hline
\end{tabular}

Tabla 9.

Efectos Olvidados de Segundo Orden

\begin{tabular}{|c|c|c|c|c|c|c|c|c|c|c|c|c|}
\hline & 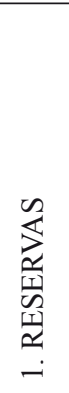 & 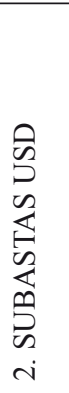 & 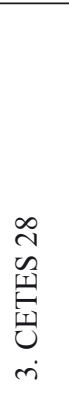 & $\begin{array}{l}U \\
\varrho \\
\dot{\sigma}\end{array}$ & 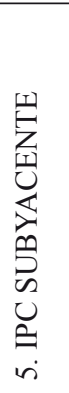 & 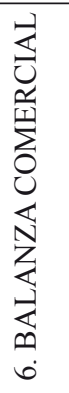 & $\begin{array}{l}\sum_{m} \\
0 \\
\Leftrightarrow \\
i\end{array}$ & 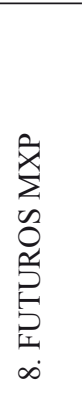 & 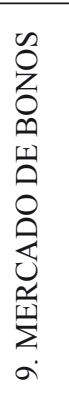 & 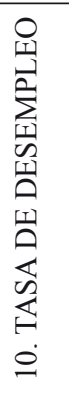 & $\begin{array}{l}\stackrel{\vartheta}{a} \\
\doteq \\
=\end{array}$ & 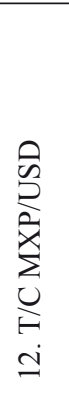 \\
\hline 1. RESERVAS & 0 & 0.6 & 0 & 0 & 0.4 & 0.1 & 0.5 & 0 & 0.1 & 0.3 & 0.2 & 0 \\
\hline 2. SUBASTAS USD & 0.3 & 0 & 0.1 & 0 & 0 & 0.4 & 0.3 & 0 & 0.1 & 0 & 0.1 & 0 \\
\hline 3. CETES 28 & 0.2 & 0.6 & 0 & 0.2 & 0.3 & 0.3 & 0.2 & 0.2 & 0 & 0.2 & 0.4 & 0.3 \\
\hline 4. IPC & 0.2 & 0 & 0.2 & 0 & 0.2 & 0.4 & 0.4 & 0.5 & 0.4 & 0.6 & 0.5 & 0.2 \\
\hline 5. IPC SUBYACENTE & 0.3 & 0 & 0.1 & 0.1 & 0 & 0 & 0.2 & 0.7 & 0.3 & 0.8 & 0.8 & 0.6 \\
\hline 6. BALANZA COMERCIAL & 0.1 & 0.5 & 0 & 0.2 & 0 & 0 & 0 & 0 & 0.1 & 0 & 0 & 0 \\
\hline 7. IPC BMV & 0 & 0.6 & 0.1 & 0.7 & 0.3 & 0.5 & 0 & 0.3 & 0 & 0 & 0 & 0 \\
\hline 8. FUTUROS MXP & 0.1 & 0.9 & 0.2 & 0.9 & 0.3 & 0.4 & 0 & 0 & 0 & 0 & 0.3 & 0.2 \\
\hline 9. MERCADO DE BONOS & 0.1 & 0 & 0 & 0 & 0.5 & 0.3 & 0 & 0.7 & 0 & 0.5 & 0.4 & 1 \\
\hline 10. TASA DE DESEMPLEO & 0.2 & 0 & 0.1 & 0.5 & 0.8 & 0.6 & 0 & 0.1 & 0.6 & 0 & 0.3 & 0.4 \\
\hline 11. PIB & 0.3 & 0 & 0.3 & 0.2 & 0.3 & 0.1 & 0 & 0.1 & 0 & 0 & 0 & 0 \\
\hline 12. T/C MXP/USD & 0.3 & 0 & 0.3 & 0.8 & 0.7 & 0 & 0.4 & 0.5 & 0.8 & 0.8 & 0.9 & 0 \\
\hline
\end{tabular}


$(5 \rightarrow 12)$ La inflación subyacente ejerce una influencia importante sobre el tipo de cambio.

Si consideramos con detalle la Tabla 8 y la Tabla 9, se observa que los expertos habían valuado el grado de incidencia de la inflación subyacente en 0.4 frente al tipo de cambio, lo que viene representando una incidencia débil. Más aún, en la valuación original, los expertos habían calculado el grado de incidencia de los bonos como nulo, cuando a través de los efectos olvidados o de segunda generación podemos apreciar que la incidencia de estos sobre el tipo de cambio es la mayor.

A partir de este ejemplo es dable asumir que, una vez que hayamos determinado los efectos que el experto se olvida, podemos asignarles un peso específico que nos permita establecer una posición de cobertura de tipo de cambio, y así evaluar su eficacia a la vez que se valida nuestra hipótesis de investigación.

\section{Radio de Cobertura de Varianza Minima (HedgeRatio)}

El ratio de cobertura o Hegde Ratio es el cociente entre el tamaño de la posición tomada en contratos de futuros sobre el tamaño del activo expuesto. Si tenemos un bien determinado y desaseamos realizar una cobertura en el mercado de futuros, se debe tomar una cantidad similar, pero en sentido opuesto en dicho mercado; entonces, hemos utilizado un ratio de cobertura de 1. Pero, si el objetivo de la persona que realiza el hedge o la cobertura es minimizar el riesgo, fijar el ratio de cobertura en 1 no es necesariamente lo óptimo.

Para reducir aún más el riesgo de la base, debemos minimizar su variabilidad, o varianza, estimando para ello el tamaño de la cobertura sobre la base de la reducción de la varianza que hemos señalado. Esto se origina al utilizar la Hedge Ratio.

Para minimizar la varianza del riesgo de la base, suponemos que esta sea:

$\delta S$ : Cambio en el precio spot, $S$, durante un período de tiempo igual a la duración de la cobertura.

$\delta F$ : Cambio en el precio del futuro, $F$, durante un período de tiempo igual a la duración de la cobertura. $\sigma s:$ Desviación estándar de $\delta S$ :

$\sigma f$ : Desviación estándar de $\delta F$ :

$\rho: \quad$ Coeficiente de correlación entre $\delta S$ y $\delta F$

$h *$ :Ratio de cobertura que minimiza la varianza de la posición del hedger.

La varianza se minimiza cuando es cero; es decir, cuando se expresa de la siguiente manera:

$$
h=\rho \quad \frac{\sigma S}{\sigma F}
$$

El ratio óptimo de cobertura es entonces el producto del coeficiente de correlación entre $S$ y $F, \rho$; y el ratio de la desviación estándar de $S$ a la desviación estándar de $F$. Si $p=1$ y $F=S$, entonces el ratio de cobertura $h$, es 1.0. Esto es normal en el caso de que el precio del futuro refleje perfectamente el precio al contado. Si $p=1$ y $\mathrm{F}=2$, el ratio de cobertura $h$, es 0.5 . Este resultado es también el esperado en el caso de que el precio del futuro cambie siempre el doble que el precio al contado.

\section{Construcción de ecuación de cobertura} (Fuzzy Hedge)

Cada una de las respuestas de la encuesta de tendencia se multiplica por las respuestas de las variables "a", "b", y "c" en la encuesta de impacto. Al terminar las multiplicaciones se suman los resultados para obtener el valor final que cae en la estrategia de cobertura, explicada posteriormente.

La Tabla 10 señala los resultados de la encuesta de tendencia donde el segundo experto respondió lo siguiente:

Tabla 10.

Resultados de la Encuesta de Tendencia

\begin{tabular}{ccc}
\hline Variable & Resultado & Valor \\
\hline A & Negativo & 0.25 \\
B & Positivo & 0.75 \\
C & Estable & 0.50 \\
\hline
\end{tabular}

En la Tabla 11 se muestra el resultado de la encuesta de impacto donde las respuestas de los expertos fueron las siguientes: 
Tabla 11.

Resultados de la Encuesta de Impacto

\begin{tabular}{cc}
\hline Variable & Valor \\
\hline A & 0.34 \\
B & 0.42 \\
C & 0.48 \\
\hline
\end{tabular}

Los resultados de ambas encuestas se usan para la ecuación de cobertura Fuzzy Hedge:

Ecuación de cobertura $=$ Fuzzy Hedge $=$ $\Sigma($ Impacto $*$ Tendencia $)$

La variable " $a$ " (impacto $*$ tendencia) + la variable " $b$ " (impacto * tendencia $)+$ la variable " $c$ ", (impacto $*$ tendencia $)=$ " $Z$ " Valor

La variable "a" 0.34(.25)+ la "b" .42(.75)

+ la "c" $0.48(.50)=0.64$ "Z" Valor

Simultáneamente se decide la cantidad de grados de cobertura que se desea obtener para la investigación, los cuales resultan al dividir la cantidad de variables estudiadas entre los grados deseados.

\section{Valor para el tipo de cobertura $=$ \\ No. de variables consideradas}

No. de grados de coberturas

En este ejemplo (Tabla 12), si se desean obtener cinco posibilidades de cobertura, se obtienen las siguientes estrategias:

Tabla 12.

Opciones de Estrategia de Cobertura

\begin{tabular}{cc}
\hline Valor para el Tipo de Cobertura & Estrategia de Cobertura \\
\hline $0 \leq \mathrm{FH}<0.60$ & $100 \%$ \\
$0.61 \leq \mathrm{FH}<1.20$ & $75 \%$ \\
$1.21 \leq \mathrm{FH}<1.80$ & $50 \%$ \\
$1.81 \leq \mathrm{FH}<2.40$ & $25 \%$ \\
$2.41 \leq \mathrm{FH}<3$ & $0 \%$ \\
\hline
\end{tabular}

Si se están estudiando tres variables y se desean las estrategias de cobertura anteriores, el procedimiento para encontrar los rangos es de 3 variables $\div 5$ estrategias, lo cual da como resultado 0.60. Este resultado de 0.60 es el que se usa para dividir los rangos de cobertura; es decir, para la "cobertura $100 \%$ " se requiere de un resultado borroso entre el 0 y el 0.60 .

Para la estrategia de "cobertura 75\%" el resultado borroso debe caer entre el 0.61 y 1.20 ; para una "cobertura de 50\%" debe caer entre el 1.21 y el 1.80; para una "cobertura de $25 \%$ " debe caer entre 1.81 y 2.40 ; y por último, el "descubierto totalmente $0 \%$ " debe caer entre el 2.41 y 3 .

Un resultado de 3 en la estrategia de "descubierto totalmente" indica que en todas las encuestas de impacto y tendencia se obtuvieron resultados perfectos; en otras palabras, la suma total de estos resultados será tres y por lo tanto no es necesaria su cobertura.

\section{Estrategia de cobertura}

La respuesta final (“Z” valor), es decir el 0.64 explicado en el paso anterior, es el resultado borroso, o Fuzzy Hedge, que se incorpora a la tabla de cobertura como resultado final, basándose en las variables estudiadas. Aplicando el ejemplo desarrollado durante los pasos anteriores, se puede observar que el Fuzzy Hedge es de 0.64 ; esto indica que se debe tener una cobertura del $75 \%$ ya que el resultado borroso se encuentra en el rango de: $0.61 \leq \mathrm{FH}<1.20$.

\section{RESULTADOS}

Para ejemplificar el método se utilizará como supuesto una empresa que tiene necesidades de financiamiento para la adquisición de maquinaria. La empresa solicita un préstamo de 10 millones de dólares. Con este préstamo, la corporación incurre en un riesgo cambiario pues la maquinaria debe liquidarse en moneda nacional (MXP), y el préstamo a un trimestre se debe pagar en dólares estadounidenses (USD).

Asumiendo este escenario, se supone que la mejor opción para la empresa es cubrirse con contratos de futuros, pues no está dispuesta a pagar una prima por una opción, y tampoco tiene operaciones en el extranjero que le otorgaría una cobertura "natural". Esto significa que la empresa tendrá que cambiar hoy 10 millones de 
dólares en el mercado spot para comprar la maquinaria en moneda nacional $\mathrm{y}$, a la vez, tendrá que cubrirse vendiendo contratos en pesos mexicanos a través del Chicago Mercantile Exchange a un trimestre, con el fin de eliminar el riesgo de apreciación del dólar .

Dentro de los supuestos se establece que se obtiene un préstamo de liquidez adicional para cumplir con el requisito del depósito de garantía de la cuenta de margen por la posición que se está tomando. Dichos fondos tendrán una tasa de interés pasiva de $7 \%$, derivado del costo financiero de los recursos. De igual forma, se instituye una tasa de interés activa de $2 \%$, como muestra del rendimiento que se está generando con dicho depósito, el mismo que se paga mediante la cámara de compensación de la bolsa de futuros en cuestión (CME).

A continuación se mencionan los supuestos para el cálculo de la cuenta de margen:

- Préstamo: 10 millones de dólares

- Tasa de interés pasiva: Libor $(5 \%)+2 \%=7 \%$

- Tasa de interés activa: $2 \%$

- Comisión por contrato: 35 dólares

- Plazo de la cobertura: 3 meses

- Valor de contrato: 500,000 pesos

- Margen inicial: 5,000 dólares

- Margen de mantenimiento: 2,500 dólares
Basados en los supuestos mencionados, se realizaron los siguientes cálculos para obtener el resultado neto de la ganancia o pérdida por la posición de cobertura para cada trimestre (ver Tabla 13).

\section{Ejemplo: Resultados Primer Trimestre 2008}

\section{Ecuación de cobertura}

$=($ Cetes $28 * 0.50)+($ IPC BMV *.075)

$+($ MexDer $($ futuros mxp $) * 0.75)+($ Política Monetaria $* 0.50)$

$+($ FX Emergentes $* 0.25)+($ FED $($ tasa de interés usa $) *$

Fuzzy Hedge = Ecuación de cobertura $=$ "Z" VALOR = 2.22

Tabla 13.

Estrategia de Cobertura: Primer Trimestre 2008

\begin{tabular}{cc}
\hline Fuzzy "Z” Valor para T/C & Estrategia de Cobertura \\
\hline $0-1.6$ & $100 \%$ \\
$1.7-3.2$ & $75 \%$ \\
$3.3-4.8$ & $50 \%$ \\
$4.9-6.4$ & $25 \%$ \\
$6.5-8$ & $0 \%$ \\
\hline
\end{tabular}

Tal como vemos en la Tabla 14a y de acuerdo al "Z" Valor obtenido en la ecuación Fuzzy Hedge, la posición de riesgo que habrá de cubrirse durante el primer trimestre del 2008 es del $75 \%$.

Tabla 14a.

Resultados Finales Cobertura 2008

\begin{tabular}{ccccc}
\hline \multirow{2}{*}{ Trimestre } & \% Cobertura & \% Cobertura & \% Cobertura & \% Cobertura \\
& Fuzzy Hedge & Hedge Ratio & Full Hedge & Full Naked \\
\hline Primero & $75 \%$ & $74.23 \%$ & $100 \%$ & $0 \%$ \\
Segundo & $75 \%$ & $80.60 \%$ & $100 \%$ & $0 \%$ \\
Tercero & $75 \%$ & $70.44 \%$ & $100 \%$ & $0 \%$ \\
Cuarto & $100 \%$ & $96.07 \%$ & $100 \%$ & $0 \%$ \\
\hline
\end{tabular}

Tabla 14b.

Resultados Finales Cobertura 2008

\begin{tabular}{lcccc}
\hline \multicolumn{1}{c}{ Trimestre } & $\begin{array}{c}\text { Ganancia/Pérdida } \\
\text { Fuzzy Hedge }\end{array}$ & $\begin{array}{c}\text { Ganancia/Pérdida } \\
\text { Hedge Ratio }\end{array}$ & $\begin{array}{c}\text { Ganancia/Pérdida } \\
\text { Full Hedge }\end{array}$ & $\begin{array}{c}\text { Ganancia/Pérdida } \\
\text { Full naked }\end{array}$ \\
\hline Primero & $-\$ 157,961.00$ & $-\$ 156,998.00$ & $-\$ 306,211.00$ & $\$ 259,859.00$ \\
Segundo & $-\$ 306,211.00$ & $-\$ 326,750.00$ & $-\$ 408,904.00$ & $\$ 382,922.00$ \\
Tercero & $\$ 259,859.00$ & $\$ 243,516.00$ & $\$ 346,479.00$ & $-\$ 371,181.00$ \\
Cuarto & $\$ 1,771,071.00$ & $\$ 1,698,946.00$ & $\$ 1,771,071.00$ & $-\$ 1,828,349.00$ \\
\hline Total & $\$ 1,566,758.00$ & $\$ 1,458,714.00$ & $\$ 1,402,435.00$ & $-\$ 1,556,749.00$ \\
\hline
\end{tabular}


Al analizar la Tabla 14b, observamos lo siguiente:

\section{A) Sin Cobertura: Full Naked}

Según se ve en la tabla mencionada, el resultado menos efectivo es el obtenido mediante la alternativa "full naked" con una pérdida acumulada de \$-1'556,749 dólares. Esta pérdida, a diferencia del resto de las operaciones de cobertura, va directamente al estado de resultados de la compañía, porque simplemente la posición de riesgo se dejó descubierta. Como consecuencia, los dólares para liquidar el pasivo tuvieron que ser recomprados al tipo de cambio final al momento del vencimiento cada trimestre.

B) Cubierto totalmente: Full Hedge

La estrategia de cobertura al 100\%, o "full hedge", arroja una utilidad acumulada anual por cobertura en contratos de futuros de \$1'402,435 dólares. Esta estrategia, al igual que la anterior, no implica una administración de riesgos como tal. Se utiliza ante períodos de alta volatilidad como el que vivimos hoy en día.

No obstante lo anterior, en esta investigación queda de manifiesto que no representa la mejor alternativa para la cobertura de riesgo de tipo de cambio de las empresas. La utilidad obtenida debe ser compensada contra la pérdida que se tendrá en el mercado spot al momento de recomprar los dólares para pagar los préstamos recibidos. En un mercado permanentemente a la alza es evidente que resulta la mejor opción, tal es el caso de los últimos dos trimestres evaluados; sin embargo, en mercados a la baja no resulta tan eficiente.

\section{C) Hedge Ratio}

El ratio de cobertura de varianza óptima obtuvo - para los cuatro trimestres - un resultado de 1'458,714 dólares positivos, convirtiéndose en la segunda mejor opción para las empresas. No obstante lo anterior, el hecho de basarse en datos históricos limita su capacidad predictiva ante situaciones de cambio en los niveles de volatilidad de los mercados, pues a mayor volatilidad, mayor ratio de cobertura. Sin embargo, al considerar datos históricos de baja volatilidad se sesga el nivel de cobertura obtenido. Si tenemos una serie de tiempo, en que el 50\% de los datos de la muestra se obtuvieron en un período de baja volatilidad y el otro $50 \%$ en períodos de alta volatilidad, la hedge ratio no podrá plasmar de manera eficiente la situación actual del mercado que se está analizando.

\section{D) Fuzzy Hedge}

La ecuación de cobertura propuesta en este ejercicio obtuvo un resultado positivo de 1'566,758 dólares. Esto la sitúa como la mejor alternativa de cobertura para las empresas que intentan administrar eficientemente su riesgo de tipo de cambio.

\section{CONCLUSIONES}

De acuerdo con los resultados obtenidos en la presente investigación se puede concluir que, siguiendo la opinión de los agentes expertos (traders, brokers) que participan en el mercado de divisas mexicano, el método ofrece una alternativa eficiente en la gestión de cobertura del riesgo cambiario sobre la evolución y el comportamiento futuro del tipo de cambio pesodólar, a través de la aplicación de un modelo no lineal de gestión de cobertura de riesgo de tipo de cambio, apoyado en la Lógica Borrosa y en la Teoría de los Efectos Olvidados. En efecto, para el período estudiado, este método produce mejores resultados que aquel que utiliza métodos de cobertura tradicional como los contratos adelantados forwards y los futuros.

Por lo que respecta al objetivo general de la investigación podemos concluir que se ha conseguido ofrecer una alternativa que puede optimizar tal proceso de cobertura. En efecto, el propósito fue construir un modelo de gestión no lineal de cobertura de riesgo cambiario que pronostique la evolución futura del tipo de cambio de equilibrio, basado en el desempeño esperado de las variables macroeconómicas que inciden en el mismo y apoyado en una variante de la Lógica Borrosa conocida como la Teoría de los Efectos Olvidados. El método se sustentó en el análisis, estudio e interpretación del comportamiento de participantes expertos (traders, brokers) en el mercado de divisas y sus reacciones ante 
cambios en las variables que inciden en el equilibrio del tipo de cambio peso-dólar. Con ello se buscó que la gestión de riesgo de tipo de cambio maximice la eficiencia y minimice el costo de una cobertura respecto de los resultados que se pueden obtener aplicando los procesos de cobertura tradicionales que existen en los mercados dedivisas.

Las interrogantes que surgieron en el desarrollo y que también constituyeron un incentivo en el desarrollo de este proyecto de investigación, podemos responder lo siguiente:

a. Sí existe evidencia sobre una relación de causalidad entre el comportamiento de las variables macroeconómicas y el tipo de cambio de equilibrio que validen una relación causa-efecto.

b. Los participantes expertos del mercado cambiario muestran tener incidencia directamente en el tipo de cambio de equilibrio, conjuntamente con el comportamiento de las variables macroeconómicas mismas.

Sobre los objetivos específicos trazados al inicio de esta investigación, es viable argumentar lo siguiente:

1. Los agentes que participan en el mercado de divisas muestran tener incidencia en el comportamiento y equilibrio de los tipos de cambio.

2. Se comprobó que las variables que, en opinión de los expertos inciden en el tipo de cambio de equilibrio, estadísticamente son propicias para ser utilizadas en el modelo de investigación toda vez que se ha comprobado su importancia y peso específico sobre el comportamiento y equilibrio del tipo de cambio peso-dólar.

3. En esta investigación se comprobó también que es factible incorporar nuevas metodologías y utilizarlas en la medición de riesgos de tipo de cambio, ya que al evaluar los resultados obtenidos se logró validar su funcionalidad en este campo de las finanzas internacionales.

Si bien nuestro modelo obtuvo los resultados que se plantearon al inicio de nuestra investigación, es importante mencionar que este modelo evidentemente es susceptible de mejorar, tarea que dejamos en manos de futuros investigadores. A continuación realizamos algunas propuestas que podrían cristalizarse en un futuro, en aras de seguir perfeccionando esta investigación, así como la administración del riesgo cambiario:

1. Buscar diferentes expertos:

- En esta investigación se fijó en un perfil específico de expertos.

- Se pueden utilizar diferentes perfiles para contrastar las características que fueron consideradas.

- Nuestro modelo fue probado con expertos que laboran en el sector financiero. Es viable utilizar ejecutivos del área de administración de riesgos y tesorería de empresas no financieras. Finalmente, este tipo de empresas no financieras se ven expuestas a riesgos de tipo de cambio día a día en su operación de negocios.

2. Considerar otras pruebas estadísticas para la validación de variables:

- En este proyecto se aplicaron las pruebas de KMO y Barlett para la validación de las variables.

- Es viable realizar la investigación con diferentes métodos estadísticos para su comprobación.

- También es posible encuestar a los expertos sobre las variables de incidencia en períodos diferentes a fin de incorporar aquellas variables que pudieron no ser consideradas e involucrarlas en el modelo, de esta manera se desecharían aquellas que pudiesen haber perdido relevancia.

3. Aplicar distintos horizontes temporales en la investigación

- La encuesta puede ser también aplicada con diferente periodicidad (semanal, semestral etc.), en función de las necesidades de la empresa.

- Esta investigación se desarrolló en cuatro trimestres; eso significa que el alcance temporal de la investigación puede ser mayor o menor dependiendo de los requerimientos específicos de quien utilice el modelo. 
4. Acotar los niveles de estrategia de cobertura:

- En esta investigación se trataron cinco diferentes niveles de estrategia de coberturas de $100 \%$, $75 \%, 50 \%, 25 \%$ y $0 \%$; por tanto, es recomendable considerar más niveles de estrategia.
5. Modelo con números borrosos unitarios, o mediante intervalos de confianza:

- En este proyecto se utilizaron números borrosos triangulares.

- Este mismo trabajo se puede realizar utilizando números unitarios o exclusivamente mediante intervalos de confianza.

\section{Referencias}

Backus, D. (1984). Empirical Models of the Exchange Rate: Separating the Wheat form de Shaff. The Canadian Journal of Economics, 17(4), 824-846.

Bazdresch, S., \& Werner, A. (2002). El comportamiento del tipo de cambio en México y el régimen de libre flotación 1996-2001. Banco de México. Documento de Investigación 9,1-18.

Bojadziev, G. M., \& Bojadziev, M. (1997). Fuzzy Logic for Business. Finance and Management. World Scientific. Recabado de <http://books.google. com.mx/books?id=a3dn0qP6q1sC\&pg=PA93\& lpg=PA93\&dq=Bojadziev, + G. + M., $+\% 26+$ Boja dziev, + M\&source $=$ bl\&ots $=2$ iL0DThd6o\&sig $=F$ o--2L3SmvGYvy8G9Oi6PddLOwI\&hl=es\#v=on epage\&q\&f=false $>$.

Cortez, K. (2004). Dinámica no lineal del tipo de cambio: aplicación al mercado mexicano. Tesis Doctoral. Universitat de Barcelona. España.

De Grauwe, P., Dewchter, H., \& Embrechts, M. (1993). Exchange Rate Theory: Chaotic Models of Foreign Exchange Markets. London: Blackwell.

Elizondo, E., \& Sepúlveda, E. (2004). Fundamentos de política cambiaria. Ejecutivos de Finanzas, 20, 36-39.

Fung, I. (2005). A Neuron-Fuzzy Computing Technique for Modeling the Time Series of Short-term Exchange Rates. Journal of American Academy of Business, September, 176.

Gil, D. F., \& Carstens, A. (1996). Some Hypothesis Related to the Mexican 1994-95 Crisis, (Documento de Investigación 9601). Consultado el 17/02/06 en $<$ www.banxico.org.mx>.
Goldberg, L., \& Tenorio, R.(1997). Strategic Trading in a Two-sided Foreign Exchange Auction. Journal of International Economics, 42, 299-326.

Gradojevic, N. (2002). Non-linear Exchange Rate Forecasting: The Role of Market Microestructure Variables. Doctoral Thesis. University of British Columbia, Vancouver, Canada.

Hull, J. (2002). Introducción a los mercados de futuros y opciones. Madrid, España: Prentice Hall.

Kaufmann A., \& Gil-Aluja, J. (1986). Introducción a la teoría de los subconjuntos borrosos para la gestión de las empresas. Santiago de Compostela, España: Milladoiro.

Kosko, B. (1995). Fuzzy Thinking, the New Science of Fuzzy Logic. New York: Hyperion Books.

Lyons, R. K., \& Evans, M. D. D. (2002). Order Flow and Exchange Rate Dynamics. Journal of Policy Economics, 110(1), 170-180.

Messe, R. A., \& Rogoff, K. (1983). Empirical Exchange Rate Models of the Seventies: Do they Fit Out of Sample? Journal of International Economics.14(1), 3-24.

Murray, R. (2005). Keeping up with World Currencies. CMA Management, 78(8), 17, ABI/INFORM Global.

Nance, D. A. (2002). Reliability and the Admissibility of Experts. Seton Hall Law Review, 34, 191-249.

Ortiz, G. (2004). The Mexican Experience under a Floating Exchange Rate Regime. Consultado 31/01/06 $<$ www.banxico.org.mx $>$. 
Rupeika-Apoga, R. (2005). Nowadays Approach to Foreign Exchange Risk Management. ABI/INFORM Global. 151: OrganizacijǿVadyba: SisteminiaiTyrimai.

Tichy, G. (2002). Over-optimism among Experts in Assessment and Foresight. Institute of TechnologyAssessment. Consultado el 19/03/08 en <http:// www.oeaw.ac.at/ita/pdef/ita_02_05.pdf $>$.

Varela, J. A. (2001). Lógica borrosa y sus aplicaciones. (ICAI, 8. Documento de la UCLM). España: Universidad de Castilla-La Mancha y la Universidad Pontificia Comillas. pp. 56-74.
Wu, I. F., \& Goo,Y. J. (2005). A Neuron Fuzzy Computing Technique for Modeling the Time Series of Short Term NT\$/US\$ Exchange Rate. Journal of American Academy of Business, 7(2), 176.

Yao, J. (1997). Spread Components and Dealer Profits in the Interbank Foreign Exchange Market. (Working paper S/98/04). New York: University Salomon Center, 1-54.

Zadeh, L. (1972). A Fuzzy-Set-Theoretic Interpretation of Linguistic Hedges. Journal of Cybernetics, 2, 4-34. 
ANEXOA

Determinación de Variables por los Expertos

\section{BANRECIO GRUPO FINANCIERO}

Prefenas

Emumaln

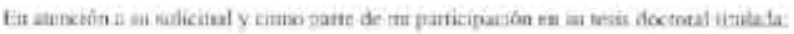

ETros Mesucano:

fa Geution de Cobertura ifel Riesge Combiarie a través de ta Teoria de los Efectos Olvidados

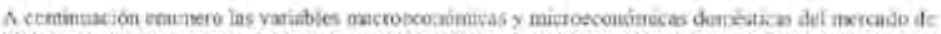

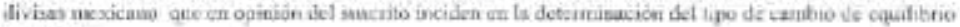
peiodily

Dictas varibbles scu las sigientes:

f- Reseryas hesthutiomeles de Bamakce

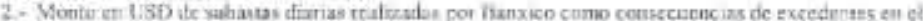
ingrelo pat vents de petrobieo.

3. Trua de ictures Coses 28 ditis

4. Irdiar Ae Preansal Cansimator

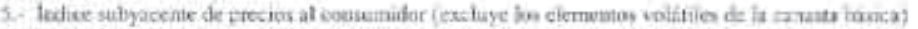

b. Mercaifa de Booos Dotnexticoe

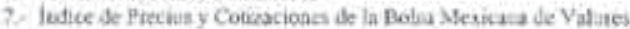

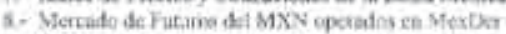

11. Dalanza Comercial

10.- Tesa de densargitro

11.-TVE

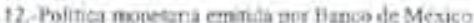

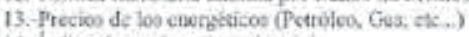

14.-Indice de prokecctain iodustria

15. Velatnea de irmesas fatriliates al pa:

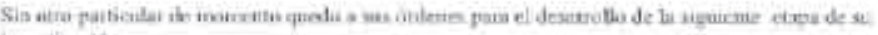
inseatigazion.

Awationite

Jove Guillagiso Shatourthorto

Reaco kegiknal de Moenrrey, S,A

Revisade de Confinmenitas

Jonge Gumalex tingane

Mise tniberisciosal Cass do Boter 


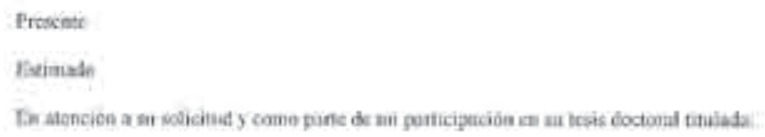

ripco Mesicanes

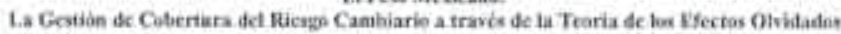

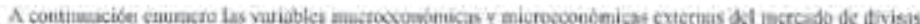

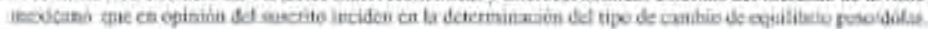

Dichas variblics sue fas seguicenes:

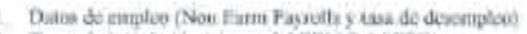

Thise de inercs (dasisiuses def FOMC Ad FED?

Mamis de la junta del rome dal fen

Beige Bool.

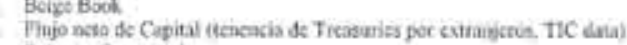

Balania Cotuctual

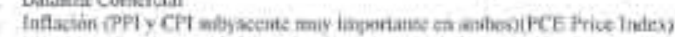

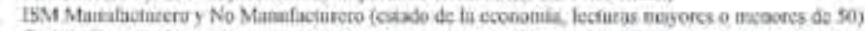

Cesita Comeme

1. Futula de ticre Derndim

12. Prodectile linhearial

12. Cinis

14. Conmmincent of trafars 1 MeM (USD,MXN)

15. Veetas dy casar micta cxirens

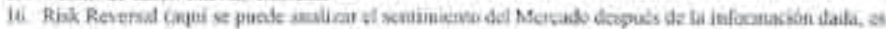

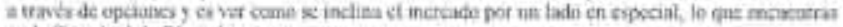
cen la Temainat de Noonibers?

17. Mereado de Pumie del MXN operitisa as CMF

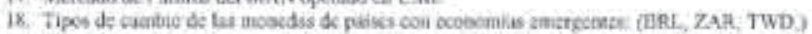

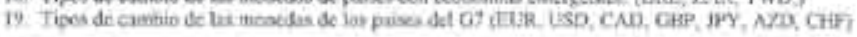

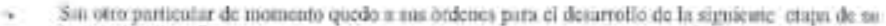
investipación
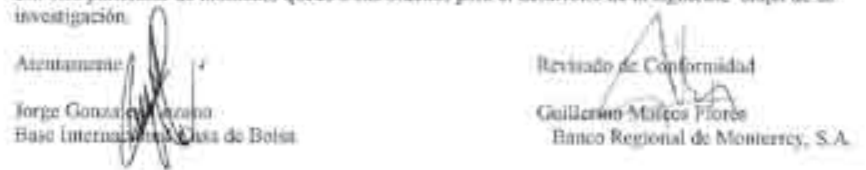

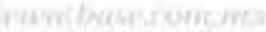

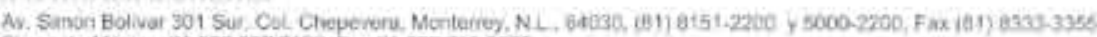

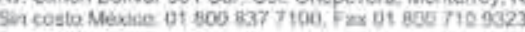




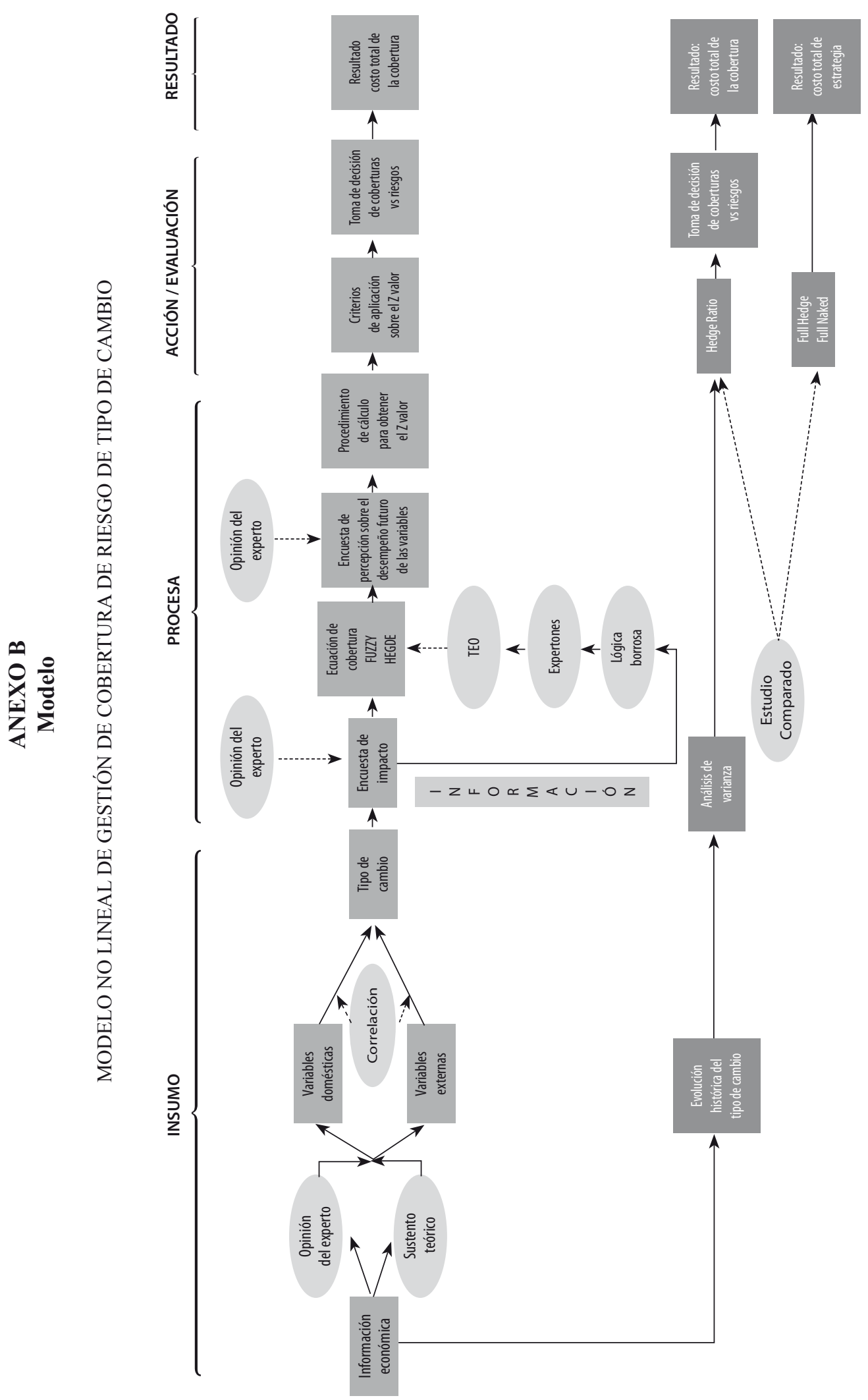

J. econ. finance adm. sci., 17(32), 2012 